\title{
Numerical Investigations of Taylor-Couette Flow Using DNS/SVV Method
}

\author{
E. Tuliszka-Sznitko* , K. Kiełczewski \\ Institute of Thermal Engineering, Poznań University of Technology \\ Poznań, ul. Piotrowo3, 60-965 Poznań \\ *E-mail: ewa.tuliszka-sznitko@put.poznan.pl
}

Received: 15 June 2015; revised: 30 September 2015; accepted: 30 September 2015; published online: 03 November 2015

\begin{abstract}
In the paper the authors present the results obtained during a numerical investigation (Direct Numerical Simulation/Spectral Vanishing Viscosity method - DNS/SVV) of a Taylor-Couette flow, i.e. the flow between two concentric disks and two concentric cylinders. The Taylor-Couette flow is one of paradigmatical systems in hydrodynamics, widely used for studying the primary instability, pattern formation, transitional flows and fully turbulent flows. Simultaneously, the flows in rotating cavities appear in numerous machines in the field of mechanics and chemistry, e.g., in cooling systems of gas turbines and axial compressors. In the paper, attention is focused on the laminar-turbulent transition region of the Taylor-Couette flow. The main purpose of the computations is to investigate the influence of different parameters (the aspect ratio, the end-wall boundary conditions, temperature gradient) on the flow structure and on flow characteristics.
\end{abstract}

Key words: Taylor-Couette flow, laminar-turbulent transition, turbulence, heat transfer, DNS

\section{INTRODUCTION}

The Taylor-Couette system (i.e. shear flow between the stationary outer cylinder and rotating inner one) provides important insight into the transitional and turbulent flows in rotating systems (Fig. 1). The results of numerical and experimental investigations of the Taylor-Couette flows are used for creating correlation formulas for the RANS method (Reynolds Averaged Navier Stokes). The RANS method is thought to be used as a basic tool in designing many industrial devices (e.g. fluid flow machineries, aircrafts, ventilation systems) for many decades to come. Pure LES (Large Eddy Simulation) and DNS (Direct Numerical Simulation) methods are very precise and deliver detailed information, but they are expensive from the numerical point of view and cannot be used for predicting various phenomena in complex industrial configurations. Additionally, the Taylor-Couette flow appears in numerous industrial installations and machines in the field of mechanics and chemistry. The results can be particularly of interest for engineers dealing with cooling systems in gas turbines and in axial compressors, ventilation installations, desalination tanks and waste water tanks, and in equipment for blood transfusion. The Taylor-Couette flow is also suitable for modelling and for predicting phenomena in geophysics and astrophysics. Due to this wide application the investigations of the Taylor-Couette flows are carried out in a number of research centers.

The experimental and numerical investigations have established that as the rotational speed of the inner cylinder increases, the fluid flow undergoes a series of bifurcations [1-7]. For a low Reynolds number a stable, laminar flow known as a cylindrical Couette flow is established. Beyond a certain critical Reynolds number, the Couette flow becomes unstable, which results in the appearance of pairs of counterrotating, axisymmetric vortices filling the annulus (TVF Taylor vortex flow). Further increase of the Reynolds numbers leads to wavy vortex flow state (referred as WVF), modulated waves state (MVF) and turbulent Taylor vortex flow (TTVF). 
However, the end-wall boundary conditions can influence this process even for a configuration of large aspect ratio $\Gamma=H /\left(R_{2}-R_{1}\right)=1 / L$. In experimental investigations, the end-walls influence the flow by constraining the axial motion of the flow and by pumping fluid along the end-walls. For the end-walls rotating with the inner cylinder, centrifugal viscous pumping causes an outflow along the end-walls. For the end-walls attached to the outer cylinder, the imbalanced pressure gradient and centrifugal forces result in an inflow along the end-walls. For the asymmetric end-wall boundary conditions a radial inflow occurs along the disk attached to the stationary outer cylinder and a radial outflow along the disk attached to the rotating inner cylinder. These Ekman layer flows determine the rotation of the nearest end-walls vortices (which appear well below the critical rotation of bifurcation to Taylor vortex flow, TVF) and subsequently determine the rotation of the entire Taylor vortex structure.

In the paper the authors report on their numerical study on the Taylor-Couette flow, performed in Poznań Supercomputing and Networking Center. Investigations are carried out by using the DNS/SVV method which allows the authors to perform computations for the higher Reynolds numbers $R e=\left(R_{2}-R_{1}\right) R_{1} \Omega / \nu$, for different thermal Rossby numbers $B=\beta\left(T_{2}-T_{1}\right)$ and for different values of geometrical parameters: aspect ratios $\Gamma=H /\left(R_{2}-R_{1}\right)=1 / L$, curvature parameter $R_{m}=\left(R_{2}+R_{1}\right) /\left(R_{2}-R_{1}\right)$ or radii ratio $\eta=\left(R_{m}-1\right) /\left(R_{m}+1\right)=R_{1} / R_{2}$. The mathematical and numerical approaches used in simulations are described in section 2 . The selected results obtained for the configurations of $\Gamma=11.75, R_{m}=19, \Gamma=3.76, R_{m}=2.2$ and $\Gamma=1.04$, $R_{m}=2.2$ are described shortly in section 3 . The summary is given in section 4 .

\section{MATHEMATICAL AND NUMERICAL APPROACHES}

The authors investigate fluid flows in a rotating cavity of the inner and outer cylinder radii $R_{1}$ and $R_{2}$, respectively (Fig. 1). The inter-disks spacing is denoted by $H$. The rotor (an inner cylinder and, in most flow cases, a bottom disk) rotates at uniform angular velocity $\Omega=\Omega e_{z}$, where $e_{z}$ is the unit vector on the $z$ axis. The flow is described by Navier-Stokes equation, continuity equation and energy equation written in a cylindrical coordinate system $(R, \varphi, Z)$ with respect to a rotating frame of reference. The Boussinesq approximation is used to take into account the buoyancy effects induced by involved body forces $\rho=\rho_{r}\left[1-\beta\left(T-T_{1}\right)\right]$, where $T_{1}$ is the temperature of cooled walls and $\beta=-1 / \rho_{r}(\partial \rho / \partial T)_{p}$. The dimensionless axial and radial coordinates are: $z=Z /(H / 2), z \in[-1,1], r=$ $\left(2 R-\left(R_{2}+R_{1}\right)\right) /\left(R_{2}-R_{1}\right), r \in[-1,1]$. The dimensionless components of the velocity vector in radial, azimuthal and axial directions are denoted by $u=U / \Omega R_{2}, v=V / \Omega R_{2}$, $w=W / \Omega R_{2}$, the dimensionless pressure is denoted by $p$, time is normalized by $\Omega^{-1}$. The dimensionless temperature is defined in the following way: $\Theta=\left(T-T_{1}\right) /\left(T_{2}-T_{1}\right)$, where $T_{2}$ is the temperature of warmed walls and $T_{1}$ is the temperature of cooled walls.

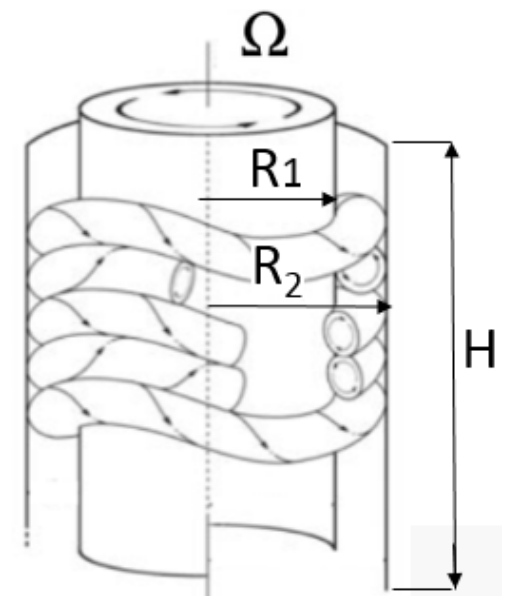

Fig. 1. Schematic picture of the Taylor-Couette flow

The governing equations, i.e. continuity equation, NavierStokes equation and energy equation in the dimensionless form are written as follows:

$$
\frac{1}{L} \frac{\partial u}{\partial r}+\frac{u}{L\left(R_{m}+r\right)}+\frac{1}{L\left(R_{m}+r\right)} \frac{\partial v}{\partial \varphi}+\frac{\partial w}{\partial z}=0,
$$

$$
\begin{aligned}
& \frac{\partial u}{\partial t}+L\left(R_{m}+1\right) A(u)= \\
= & -\left(R_{m}+1\right) \frac{\partial p}{\partial r}+\frac{L^{2}\left(R_{m}-1\right) 2}{R e} \\
\times & {\left[\nabla^{2} u-\frac{u}{L^{2}\left(R_{m}+r\right)^{2}}-\frac{2}{L^{2}\left(R_{m}+r\right)^{2}} \frac{\partial v}{\partial \varphi}\right], }
\end{aligned}
$$

$$
\begin{aligned}
& \frac{\partial v}{\partial t}+L\left(R_{m}+1\right) A(v)= \\
= & -\frac{\left(R_{m}+1\right)}{\left(R_{m}+r\right)} \frac{\partial p}{\partial \varphi}+\frac{L^{2}\left(R_{m}-1\right) 2}{R e} \\
\times & {\left[\nabla^{2} v-\frac{v}{L^{2}\left(R_{m}+r\right)^{2}}+\frac{2}{L^{2}\left(R_{m}+r\right)^{2}} \frac{\partial u}{\partial \varphi}\right], } \\
& \frac{\partial w}{\partial t}+L\left(R_{m}+1\right) A(w)=-L\left(R_{m}+1\right) \frac{\partial p}{\partial z} \\
+ & \frac{L^{2}\left(R_{m}-1\right) 2}{R e}\left(\nabla^{2} w\right),
\end{aligned}
$$

$$
\frac{\partial \Theta}{\partial t}+L\left(R_{m}+1\right) A(\Theta)=\frac{L^{2}\left(R_{m}-1\right) 2}{\operatorname{Re} \operatorname{Pr}}\left(\nabla^{2} \Theta\right),
$$


where:

$$
\begin{gathered}
A(u)=\frac{1}{L} u \frac{\partial u}{\partial r}+\frac{v}{L\left(R_{m}+r\right)} \frac{\partial u}{\partial \varphi}+w \frac{\partial u}{\partial z}-(1-B \Theta) \\
\times\left[\frac{v^{2}}{L\left(R_{m}+r\right)}+\frac{2 v}{L\left(R_{m}+1\right)}+\frac{\left(R_{m}+r\right)}{L\left(R_{m}+1\right)^{2}}\right] \\
A(v)=\frac{1}{L} u \frac{\partial v}{\partial r}+\frac{v}{L\left(R_{m}+r\right)} \frac{\partial v}{\partial \varphi}+w \frac{\partial v}{\partial z} \\
+(1-B \Theta)\left[\frac{u v}{L\left(R_{m}+r\right)}+\frac{2 u}{L\left(R_{m}+1\right)}\right] \\
A(w)=\frac{1}{L} u \frac{\partial w}{\partial r}+\frac{v}{L\left(R_{m}+r\right)} \frac{\partial w}{\partial \varphi}+w \frac{\partial w}{\partial z} \\
A(\Theta)=\frac{1}{L} u \frac{\partial \Theta}{\partial r}+\frac{1}{L\left(R_{m}+r\right)} v \frac{\partial \Theta}{\partial \varphi}+w \frac{\partial \Theta}{\partial z}
\end{gathered}
$$

No-slip boundary conditions are applied to all rigid walls $u=w=0$. For the azimuthal velocity component, the boundary conditions are: $v=0$ on the rotating walls and $v=-\left(R_{m}+r\right) /\left(R_{m}+1\right)$ on the stationary walls. The temperature boundary conditions are as follows: $\Theta=0$ on the cooled walls and $\Theta=1$ on the warmed walls. Prandtl number is equalled 0.71 .

All presented results are obtained with the use of the DNS/SVV method. The spatial scheme is based on a pseudospectral Chebyshev-Fourier-Galerkin collocation approximation:

$$
\begin{aligned}
\Psi(r, \varphi, z) & =\sum_{k=-N^{\varphi} / 2}^{N^{\varphi} / 2-1} \sum_{n=0}^{N^{r}-1} \sum_{m=0}^{N^{z}-1} \hat{\Psi}_{n m k} T_{n}(r) T_{m}(z) e^{i k \varphi}, \\
& -1 \leq r, z \leq 1, \quad 0 \leq \varphi \leq 2 \pi .
\end{aligned}
$$

Where $T_{n}(r)$ and $T_{m}(z)$ are Chebyshev polynomials of degrees $n$ and $m, N^{r}, N^{\varphi}$ and $N^{z}$ are numbers of collocation points in radial, azimuthal and axial directions, respectively. The temporal approximation used in the paper is a projection scheme, based on backward differentiation in time. This scheme combines an implicit treatment of the diffusive term and an explicit Adams-Bashforth scheme for the non-linear convective terms. At every time iteration $t^{(n+1)}$ the computations start with obtaining pressure predictor $p^{p}$ by solving the Poisson equation

$$
\begin{aligned}
& \frac{1}{L^{2}} \frac{\partial^{2} p^{p}}{\partial r^{2}}+\frac{1}{L^{2}\left(R_{m}+r\right)} \frac{\partial p^{p}}{\partial r}+\frac{1}{L^{2}\left(R_{m}+r\right)^{2}} \frac{\partial^{2} p^{p}}{\partial \varphi^{2}} \\
+ & \frac{\partial^{2} p^{p}}{\partial z^{2}}=\operatorname{Div}\left[-2 A(V)^{n}+A(V)^{n-1}\right]
\end{aligned}
$$

with Neumann boundary conditions. In the above equation $(n)$ and $(n-1)$ depict the previous iterations. Then, predictors of the velocity components $u^{p}, v^{p}, w^{p}$ are obtained by solving the Helmholtz equations with appropriate boundary conditions. For instance, for $r$ direction equation is written in the following form:

$$
\begin{aligned}
& \nabla^{2} u^{p}-u^{p} \frac{1}{L^{2}\left(R_{m}+r\right)^{2}}-\frac{R e}{L^{2} 2\left(R_{m}-1\right)} \frac{3}{2} \frac{u^{p}}{(\delta t)}= \\
= & \frac{R e}{L^{2}\left(R_{m}-1\right) 2} \times\left\{\frac{-4 u^{n}+u^{n-1}}{2(\delta t)}\right. \\
+ & \left.L\left(R_{m}+1\right)\left[2 A(u)^{n}-A(u)^{n-1}\right]+\left(R_{m}+1\right) \frac{\partial p^{p}}{\partial r}\right\} \\
+ & \frac{2}{L^{2}\left(R_{m}+r\right)^{2}}\left(2 \frac{\partial v^{n}}{\partial \varphi}-\frac{\partial v^{n-1}}{\partial \varphi}\right) .
\end{aligned}
$$

The correction of the velocity field is done by taking into account the pressure gradient at iteration $t^{n+1}$, so that the final velocity field satisfies the incompressibility constraint

$$
\begin{aligned}
3\left(V^{n+1}-V^{p}\right) /\left[L\left(R_{m}+1\right) 2 \delta t\right] & = \\
=-\left(\nabla p^{n+1}-\nabla p^{p}\right), \quad \nabla \cdot V^{n+1} & =0
\end{aligned}
$$

with the boundary condition $V^{n+1} \cdot n=V^{p} \cdot n$. Corrected velocity components are obtained by computing new variable $\phi=2 \delta t\left(p^{n+1}-p^{p}\right) / 3$ from the equation $\nabla \phi=$ $\operatorname{div}\left(V^{p}\right) /\left[L\left(R_{m}+1\right)\right]$ with boundary condition $\nabla(\phi) \cdot n=0$. All unknown variables $\Psi=\left[u^{p}, v^{p}, w^{p}, p^{p}, \Theta, \phi\right]^{T}$ are obtained by solving the Helmholtz equation (5):

$$
\begin{aligned}
& \frac{1}{L^{2}} \frac{\partial^{2} \Psi}{\partial r^{2}}+\frac{1}{L^{2}\left(R_{m}+r\right)} \frac{\partial \Psi}{\partial r} \\
+ & \frac{1}{L^{2}\left(R_{m}+r\right)^{2}} \frac{\partial^{2} \Psi}{\partial \varphi^{2}}+\frac{\partial^{2} \Psi}{\partial z^{2}}-q \Psi=S .
\end{aligned}
$$

Where $q$ is a constant or is a function of $r$. Function $S$ contains terms from the previous iterations and from the predictor stage. In the next step the equation (5) is extended to Fourier series:

$$
\begin{aligned}
& \quad \frac{1}{L^{2}} \frac{\partial^{2} \hat{\Psi}_{k}}{\partial r^{2}}+\frac{1}{L^{2}\left(R_{m}+r\right)} \frac{\partial \hat{\Psi}_{k}}{\partial r}+\frac{\partial^{2} \hat{\Psi}_{k}}{\partial z^{2}} \\
& -\left[q+\frac{k^{2}}{L^{2}\left(R_{m}+r\right)^{2}}\right] \hat{\Psi}_{k}=\hat{S}_{k}, \\
& \quad k \in\left[-N^{\varphi} / 2, \ldots, N^{\varphi} / 2-1\right] .
\end{aligned}
$$

After spatial discretization in radial and axial direction equation (6) can be written in the following form:

$$
\begin{aligned}
& A \Psi+\Psi B=S, \quad \Psi=\hat{\Psi}_{i j k}=\hat{\Psi}_{k}\left(r_{i}, z_{j}\right), \\
& S=\hat{S}_{i j k}=\hat{S}_{k}\left(r_{i}, z_{j}\right), \quad-1 \leq r_{i}, z_{j} \leq 1 .
\end{aligned}
$$

where:

$$
\begin{gathered}
A_{i j}=\frac{1}{L^{2}} D r_{i j}^{(2)}+\frac{1}{L^{2}\left(R_{m}+r_{i}\right)} D r_{i j}^{(1)} \\
-\left[q_{i}+\frac{k^{2}}{L^{2}\left(R_{m}+r_{i}\right)^{2}}\right] \delta_{i j}, \quad B_{i j}=D z_{j i}^{(2)} .
\end{gathered}
$$

$D r_{i j}^{(1)}, D r_{i j}^{(2)}, D z_{i j}^{(2)}$ are differentiating matrices. The final system of equations is solved with full diagonalization. 
To stabilize computations for the higher Reynolds numbers the authors use the SVV method proposed by Tadmor [11], in which an artificial viscous operator is added to Laplace operator. The one-dimensional modified Laplace operator can be written in the following form:

$$
\nu \Delta^{S V V} u_{N}=\nu \Delta u_{N}+\varepsilon_{N} \partial\left(Q_{N} \partial u_{N} / \partial x\right) / \partial x .
$$

In equation (9a) $u_{N}$ is a discrete approximation of velocity component, $\varepsilon_{N}$ is the viscosity amplitude. $Q_{N}$ is the spatial operator (active for higher frequencies) which in Fourier space can be written as follows:

$$
\begin{aligned}
& \varepsilon_{N} \partial\left(Q_{N} \partial u_{N} / \partial x\right) / \partial x= \\
= & -\varepsilon_{N} \sum_{k_{T} \leq|k| \leq N / 2} k^{2} \hat{Q}_{N / 2}(k) \hat{u}_{k} e^{i k x} .
\end{aligned}
$$

In Chebyshev space:

$$
Q_{N}\left(\partial u_{N} / \partial x\right)=\sum_{k=k_{T}}^{N} \hat{Q}_{N}(k)\left(\partial u_{N} / \partial x\right) T_{k} .
$$

where $T_{k}$ are Chebyshev polynomials. In equations (9b) and (9c) $\hat{Q}_{N}$ is defined in the following way:

$$
\hat{Q}_{N}(k)=\left\{\begin{array}{ll}
0 & \text { for } 0 \leq|k| \leq k_{T} \\
\exp \left[-\left(\frac{N-|k|}{k_{T}-|k|}\right)^{2}\right] & \text { for } k_{T}<|k| \leq N
\end{array},\right.
$$

where $k_{T}$ is the threshold mode above which SVV operator is active. The modified 3D Laplace operator takes the form, $[12,13]$ :

$$
\begin{aligned}
\nu \Delta^{S V V} u_{N} & \equiv \nu \Delta u_{N}+\nabla \cdot\left(\varepsilon_{N} Q_{N} \cdot\left(\nabla u_{N}\right)\right)= \\
& =\nu\left(\nabla \cdot G_{N} \cdot \nabla\right) u_{N}
\end{aligned}
$$

where

$$
\begin{aligned}
& G_{N}=I+\frac{1}{\nu} \varepsilon_{N} Q_{N}= \\
= & {\left[\begin{array}{ccc}
1+\varepsilon_{N^{r}}^{r} Q_{N^{r}}^{r} / \nu & 0 & 0 \\
0 & 1+\varepsilon_{N^{\varphi}}^{\varphi} Q_{N^{\varphi}}^{\varphi} / \nu & 0 \\
0 & 0 & 1+\varepsilon_{N^{z}}^{z} Q_{N^{z}}^{z} / \nu
\end{array}\right] } \\
= & {\left[\begin{array}{ccc}
G_{N^{r}}^{r} & 0 & 0 \\
0 & G_{N^{\varphi}}^{\varphi} & 0 \\
0 & 0 & G_{N^{z}}^{z}
\end{array}\right] . }
\end{aligned}
$$

In equations (10a) and (10b) $\nabla u_{N}$ is the velocity gradient tensor, $\varepsilon_{N^{i}}^{i}$ is a viscosity amplitude for $i$ direction. The modified Laplace operator can be written as follows:

$$
\begin{gathered}
\Delta^{S V V} \equiv \frac{1}{L^{2}} \frac{\partial}{\partial r} G_{N^{r}}^{r} \frac{\partial}{\partial r}+\frac{1}{L^{2}\left(R_{m}+r\right)} G_{N^{r}}^{r} \frac{\partial}{\partial r} \\
+\frac{1}{L^{2}\left(R_{m}+r\right)^{2}} \frac{\partial}{\partial \varphi} G_{N^{\varphi}}^{\varphi} \frac{\partial}{\partial \varphi}+\frac{\partial}{\partial z} G_{N^{z}}^{z} \frac{\partial}{\partial z} .
\end{gathered}
$$

After expanding equation (11) in Fourier series, the Laplace operator takes the form:

$$
\begin{aligned}
& \Delta_{k}^{S V V}=\frac{1}{L^{2}} \frac{\partial}{\partial r} G_{N^{r}}^{r} \frac{\partial}{\partial r}+\frac{1}{L^{2}\left(R_{m}+r\right)} G_{N^{r}}^{r} \frac{\partial}{\partial r} \\
& -\frac{k^{2}}{L^{2}\left(R_{m}+r\right)^{2}} \hat{G}_{N^{\varphi}}^{\varphi}(k)+\frac{\partial}{\partial z} G_{N^{z}}^{z} \frac{\partial}{\partial z} \\
& \quad k \in\left[-N^{\varphi} / 2, \ldots, N^{\varphi} / 2-1\right] .
\end{aligned}
$$

In SVV method matrices $A_{i j}$ and $B_{i j}$ (equation (8)) take the forms:

$$
\begin{gathered}
A_{i j}=\frac{1}{L^{2}} D r_{i j}^{(2) S V V}+\frac{1}{L^{2}\left(R_{m}+r\right)} D r_{i j}^{(1) S V V} \\
-\left(q_{i}+\frac{k^{2}}{L^{2}\left(R_{m}+r\right)^{2}}\left(1+\frac{\varepsilon_{N^{\varphi}}^{\varphi}}{\nu} \hat{Q}_{N^{\varphi} / 2}^{\varphi}(k)\right)\right) \delta_{i j},
\end{gathered}
$$

$$
B_{i j}=D z_{j i}^{(1) S V V} .
$$

The differentiating matrices $D r_{i j}^{(1) S V V}$ and $D r_{i j}^{(2) S V V}$ are defined as follows:

$$
\left[D r_{i j}^{(2) S V V}\right]=D r^{(2) S V V}=D r^{(1)} \cdot D r^{(1) S V V}
$$

$$
\left[D r_{i j}^{(1) S V V}\right]=D r^{(1) S V V}=\left(I+\frac{1}{\nu} \varepsilon_{N^{r}}^{r} H^{r}\right) D r^{(1)} .
$$

The differentiating matrix $D z_{i j}^{(1) S V V}$ is defined in a similar way. In the above equation $H^{r}$ is Chebyshev high-pass filter [14] that can be written in $r$ direction in the following way:

$$
\begin{gathered}
H_{i j}^{r}=\frac{2}{N^{r} c_{j}} \sum_{n=0}^{N^{r}-1} \frac{1}{c_{n}} \hat{Q}_{N^{r}}^{r}(n) T_{n}\left(r_{i}\right) T_{n}\left(r_{j}\right) \\
c_{j}= \begin{cases}2 & j \in\left(0, N^{r}\right) \\
1 & j \in\left(1, \ldots, N^{r}-1\right),\end{cases} \\
T_{n}\left(r_{j}\right)=\cos \left(n \pi(j) / N^{r}\right) .
\end{gathered}
$$

The presented algorithm turns out to be a very effective tool for investigating transitional and turbulent flows. More detailed information can be found in [12, 13]. 


\section{SELECTED RESULTS}

The authors' investigations of Taylor-Couette flows are focused on determining the influence of geometrical parameters such as aspect ratio $\Gamma$, curvature parameter $R_{m}$, Reynolds number, the thermal Rossby number and boundary conditions on the flow structure and on statistical values. The statistical results are prepared in the form of axial and radial profiles of averaged (over time and azimuthal direction) three velocity components, torque, components of the Reynolds stress tensor and many structural parameters, which are useful for modelling purposes. Visualization is one of the most important aspects in analysis of the flow structures. From the identification techniques based on pointwise analysis of the velocity gradient tensor $(Q$ criterion, $\Delta$ criterion, $\omega$ criterion, $\lambda_{2}$ criterion, [19-20]) the authors chose the $\lambda_{2}$ criterion, which represents the topology of vortex cores correctly. The $\lambda_{2}$ criterion captures the regions of local pressure minimum. The background of vortex identification schemes can be found in [19-20]. The iso-surfaces of $\lambda_{2}$ (the second, in magnitude, eigenvalue of the tensor $S_{i k} S_{k j}+\Omega_{i k} \Omega_{k j}$, where $S_{i j}=\left(\partial u_{i} / \partial x_{j}+\partial u_{j} / \partial x_{i}\right) / 2$, $\left.\Omega_{i j}=\left(\partial u_{i} / \partial x_{j}-\partial u_{j} / \partial x_{i}\right) / 2\right)$ show perfectly the flow structure in three-dimensional space. In the section the authors present their exemplary numerical results obtained using mesh up 12 million collocation points.

The authors have started their research of the TaylorCouette flow from the investigations of the flow in the configuration $\Gamma=11.75, R_{m}=19.0$ with stationary outer cylinder and rotating inner one (the results obtained for the lower $R e$ were published in [15]). The computations have been performed for the symmetric (both disks stationary) and the asymmetric end-wall boundary conditions (the upper disk stationary and the bottom one rotating). In Fig. 2 the flow structure obtained for the asymmetric end-wall boundary conditions is presented: the flow is pumped radially outwards along the rotating bottom disk and propagates inwards along the stationary top disk. It is visible from Fig. 2 that the flow near the bottom disk is more disturbed than the flow near the top one. For the symmetric end-wall boundary conditions the flow propagates inwards along both disks.

The study of the flow case $\Gamma=11.75, R_{m}=19.0$ has allowed for the analysis of all the subsequent bifurcations which appear with the increasing Reynolds number. For both symmetric and asymmetric end-wall boundary conditions the Taylor-Couette vortices (TVF) are formulated slightly above $R e=80$ and the transition to unsteadiness takes place at $R e=200$, which is manifested by the appearance of wavy vortex flow (WVF). The modulated wavy vortex flow (MVF) obtained at $R e=1161$ is presented in Fig. 2a (the

b)
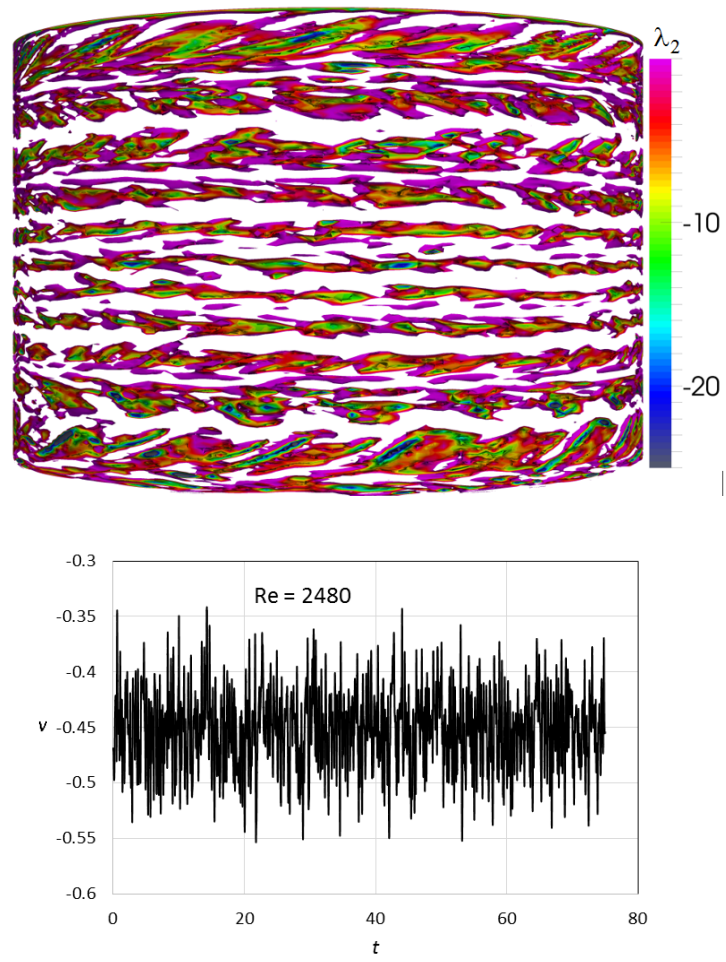

Fig. 2. The iso-surfaces of instantaneous $\lambda_{2}$ and instability characteristics obtained for: a) $\operatorname{Re}=1161$, b) $R e=2480$. $\Gamma=11.75$, $R_{m}=19$, the asymmetric end-wall boundary conditions 
iso-surfaces of $\lambda_{2}$ obtained over cylindrical surface near the inner cylinder). Together with the graphs showing the isosurfaces of $\lambda_{2}$ the authors present the instability characteristics $(v(t)$ azimuthal velocity component as a function of time) obtained at the collocation point ( $r=-0.8, z=0$, $\varphi=0$ ). From the characteristics obtained for $R e=1161$ it is visible that on the fundamental wave (of angular frequency $\sigma=2 \pi / \Delta t=5.2$, where $\Delta t$ is dimensionless period of oscillation) the second wave of a much lower frequency (of angular frequency $\sigma=2 \pi / \Delta t=0.25$ ) is superimposed. The turbulent Taylor vortex flows (TTVF) obtained for $R e=2480$ is presented in Fig. 2b. In Fig. 2b there are many small-scale vortical structures extended along the azimuthal direction over the cylindrical surface near the inner cylinder, [5]. The instability characteristic obtained for $R e=2480$ is chaotic. The instability characteristics obtained for the symmetric and asymmetric end-wall boundary conditions differ in some aspects (the difference in frequencies has been observed), but generally for as high aspect ratios as $\Gamma=11.75$ the influence of the end-walls is small.

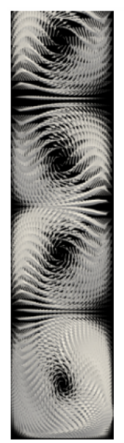

$\operatorname{Re}=160$

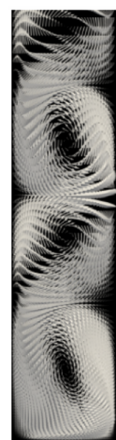

450

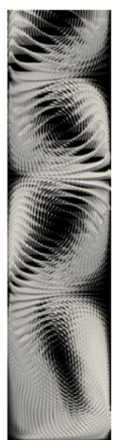

981

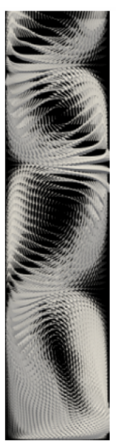

1490

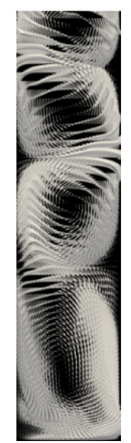

2120

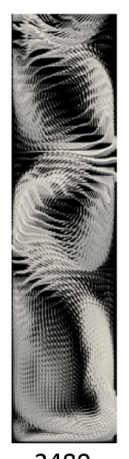

2480
Fig. 3. The instantaneous meridian flows obtained for the different Reynolds numbers (only the area near the bottom rotating disk is visible). $\Gamma=11.75, R_{m}=19$, the asymmetric end-wall boundary conditions, the inner cylinder is on the left

For the Reynolds numbers from the range $R e=160 \div$ 2480 in Fig. 3 the snapshots of instantaneous velocity fields in a radial-axial plane are presented. In Fig. 3 the authors present only the lower part of cavity near the bottom rotating disk in order to show in detail this part of the meridian flow. It is visible in Fig. 3 that for all considered Reynolds numbers from the range $R e=160 \div 2480$ the large-scale Taylor vortices occupy the entire gap, with well-defined inflow and outflow jets. However, as the Reynolds number increases, the Taylor vortices become gradually distorted.

The authors have continued the study of the TaylorCouette flow with the asymmetric end-wall boundary conditions for configurations of smaller aspect ratio: $\Gamma=3.76$, $R_{m}=2.2$. For a configuration of aspect ratio $\Gamma=3.76$, $R_{m}=2.2$ with a rotating inner cylinder and bottom disk, and with a stationary outer cylinder and top disk the sequence of events occurring with the increasing $R e$ is as follows: The three-cell structure is fully formulated at Reynolds number $R e=77$ (the odd number of vortices is a characteristic feature of the Taylor-Couette flow with the asymmetric end-wall boundary conditions). Fig. 4a, 4b and 4c show the meridian flows obtained for the different Reynolds numbers.

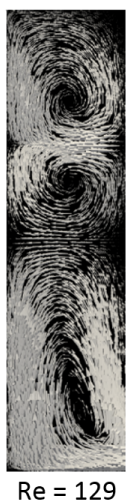

a)

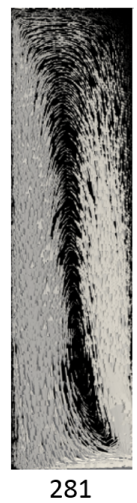

b)

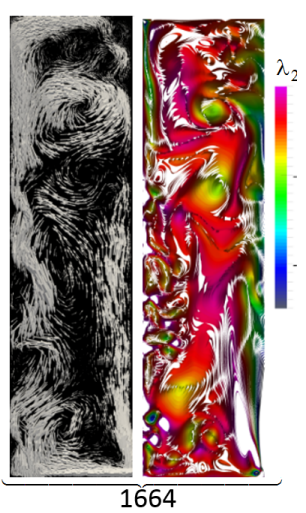

c)

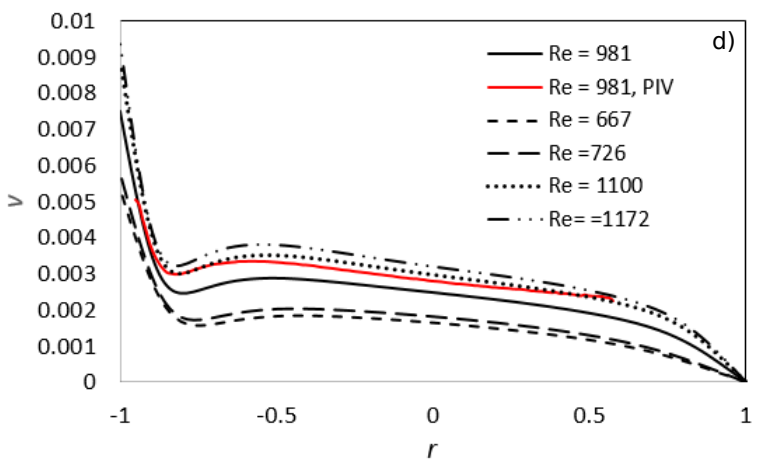

Fig. 4. a) The meridian flow, $R e=129$, b) the meridian flow, $R e=281, \mathrm{c})$ the meridian flow and the iso-surfaces $\left(0^{\circ} \leq \varphi \leq 2^{\circ}\right)$ of $\left.\lambda_{2}, R e=1664, \mathrm{~d}\right)$ the radial profiles of the azimuthal velocity component, comparison of the experimental PIV results and the numerical data. $\Gamma=3.76, R_{m}=2.2$

It is visible in Fig. 4a that there is an inward flow towards the rotating inner cylinder (on the left) along the stationary top disk and an outward flow along the rotating disk. At slightly larger $R e$ (about 270) the middle vortex is squeezed by the growth of the top and bottom vortices, and finally at $R e=281$ the transition from the steady three-cell structure to a steady one cell-structure takes place (Fig. 4b). It is visible in Fig. $4 \mathrm{~b}$ that fluid is pumped radially outwards along the rotating disk, then it is transported up along the stationary outer cylinder and recirculates along the upper disk. Finally, the fluid is transported downward along the rotating inner cylinder towards the rotating disk. The transition to unsteadiness for cavity of aspect ratio $\Gamma=3.76$ and $R_{m}=2.2$ occurs at $R e=492$. Fig. $4 \mathrm{c}$ shows the meridian flow and the 3D flow structures (iso-surfaces of $\lambda_{2}$ ) obtained for $R e=1664$. From Fig. $4 \mathrm{c}$ it is visible that the most disturbed areas in this 


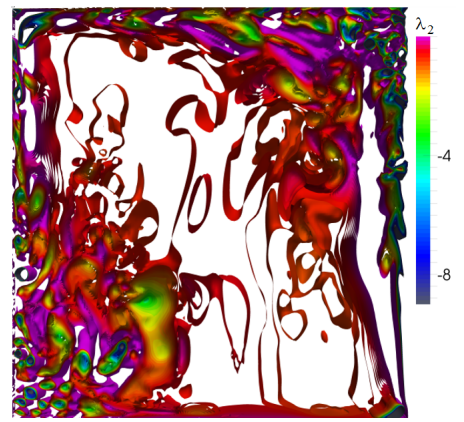

a)

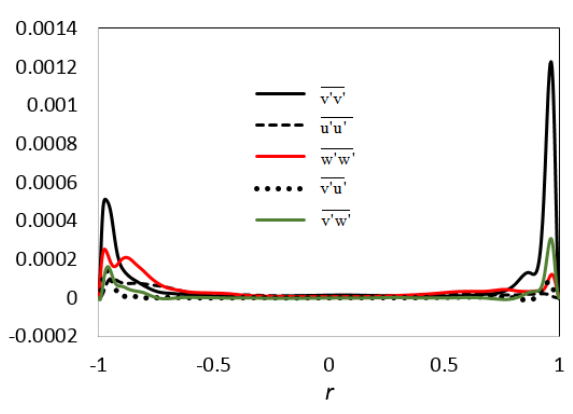

b)

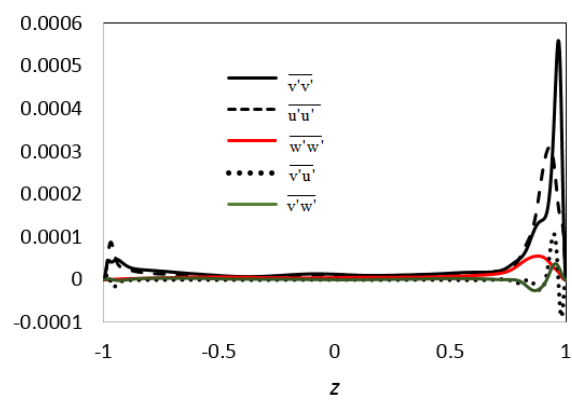

C)

Fig. 5. a) The iso-surfaces $\left(0^{\circ} \leq \varphi \leq 2^{\circ}\right)$ of $\lambda_{2}$ showing structure of the meridian flow, b) the radial profiles of $\overline{v^{\prime} v^{\prime}}, \overline{u^{\prime} u^{\prime}}, \overline{w^{\prime} w^{\prime}}, \overline{v^{\prime} u^{\prime}}, \overline{v^{\prime} w^{\prime}}$ obtained in the middle section $z=0$, c) the axial profiles of $\overline{v^{\prime} v^{\prime}}, \overline{u^{\prime} u^{\prime}}$ obtained in the middle section $r=0 . \Gamma=1.04, R_{m}=2.2, R e=15234$

flow case are located near the inner rotating cylinder. The authors have studied the flow case of $\Gamma=3.76, R_{m}=2.2$ also experimentally in the frame of EuHIT project in Brandenburg University of Technology in cooperation with prof. Ch. Egbers, prof. U. Harlander and their Colleagues. An exemplary radial profile of the azimuthal velocity component obtained using PIV measurements (Particle Image Velocimetry) in axial section $z=0.827$ (section near the top stationary disk) is presented in Fig. 4d together with the profiles obtained numerically by the authors for different $R e$. This comparison shows agreement between experimental and numerical results (more detailed comparison between experimental and numerical results will be presented during ICTW2015 workshop in Cottbus and ETC2015 in Delft). The authors' research on the influence of the end-wall boundary conditions on the flow structure and on transition from a three-cell structure to a one-cell structure is the extension of the study performed in papers [16-18], which were conducted only for the low Reynolds numbers.

The authors have also performed computations of the flow structure in the cavities of aspect ratio close to one: $\Gamma=1.04, R_{m}=2.2$ and $\Gamma=1.47, R_{m}=2.2$ with the asymmetric end-wall boundary conditions. With the asymmetric end-wall boundary conditions a one-cell structure is observed in both configurations $\left(\Gamma=1.04, R_{m}=2.2\right.$ and $\left.\Gamma=1.47, R_{m}=2.2\right)$. An exemplary meridian flow $\left(\lambda_{2}\right.$ isosurfaces) obtained numerically for $R e=15234$ ( $\Gamma=1.04$, $R_{m}=2.2$ ) is presented in Fig. 5a. It is visible in Fig. 5a that the maximum of turbulence is located at the junction between the stationary outer cylinder and the stationary top disk. However, the area at the corner between the inner cylinder and the rotating bottom disk is also highly disturbed by the vortices propagating downwards along the inner cylinder. The remaining part of the rotating disk boundary layer is fully laminar. The most disturbed areas are also visible in Figures $5 \mathrm{~b}$ and $5 \mathrm{c}$ where the radial and axial profiles of the Reynolds stress tensor components $\overline{v^{\prime} v^{\prime}}, \overline{u^{\prime} u^{\prime}}, \overline{w^{\prime} w^{\prime}}, \overline{v^{\prime} u^{\prime}}, \overline{v^{\prime} w^{\prime}}$ are presented respectively (the profiles are obtained in the middle sections of cavity, for $z=0$ and $r=0$ ). The radial profile of the azimuthal Reynolds stress tensor component $\overline{v^{\prime} v^{\prime}}$ obtained for $z=0$ shows a very rapid increase of $\overline{v^{\prime} v^{\prime}}$ near the outer stationary cylinder and slightly lower peak near the inner cylinder (values $\overline{u^{\prime} u^{\prime}}, \overline{v^{\prime} u^{\prime}}, \overline{v^{\prime} w^{\prime}}$ are one order smaller). The axial profiles of $\overline{v^{\prime} v^{\prime}}$ and $\overline{u^{\prime} u^{\prime}}(r=0)$ show maximum values in the stator boundary layer, and very small values in the rotor boundary layer. The similar profiles of the Reynolds stress tensor components have been obtained for the configuration of $\Gamma=1.47$. For the flow case $\Gamma=1.04, R_{m}=2.2$ the authors have also performed experimental investigations in the frame of EuHIT using PIV and Kalliroscopy methods and they have found good agreement between results (see Fig. 6).

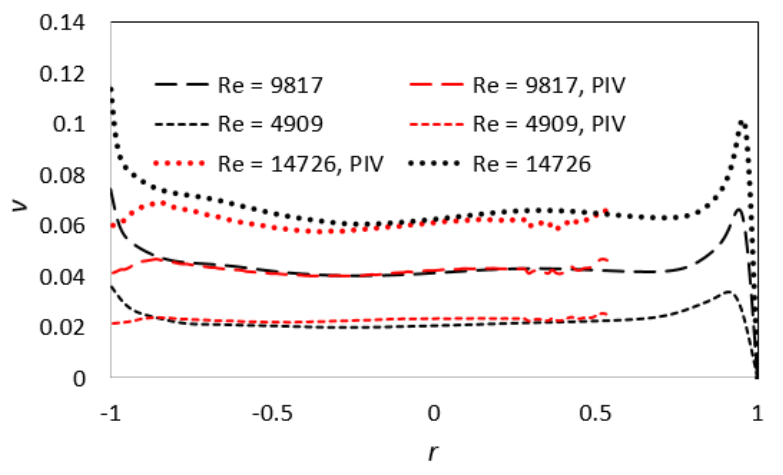

Fig. 6. The radial profiles of the azimuthal velocity component obtained in axial sections $z=-0.542$ for the different Reynolds numbers, comparison with the PIV results. $\Gamma=1.04, R_{m}=2.2$

The configuration of aspect ratio $\Gamma=3.76$ is also used by the authors to check the influence of the curvature parameter $R_{m}$ and the thermal conditions on the flow structure and statistical parameters. The preliminary results obtained by the authors for non-isothermal conditions $\left(\Gamma=20, R_{m}=3\right)$ were published in [15]. The main purpose is to deliver distributions of the Nusselt numbers along cylinders and disks, 
which can be particularly interesting for engineers dealing with cooling systems. Another issue investigated numerically with the use of the computer rescourses of Poznan Supercomputing and Networking Center is the influence of the periodic modulation (superimposed on the inner cylinder rotational speed) on the instability critical parameters and on the flow structure. The results can answer the questions which concerns the degrees of stabilization that can be achieved using periodic modulation. This issue is important for controlling 3D boundary layers.

\section{SUMMARY}

In the paper the authors have presented the main scientific problems which they have investigated numerically with the use of computational resources of Poznań Supercomputing and Networking Center. The main purpose is to explore the phenomena occurring in the Taylor-Couette flows for the transitional Reynolds numbers and deliver statistical data which can be used for creating new models for the RANS method. The selected results obtained during numerical investigations of the Taylor-Couette flows have been presented. The authors have focused on the three chosen flow cases. The first one $\Gamma=11.75, R_{m}=19$ has allowed the authors to analyze the flow structures which appear with the increasing Reynolds number. All consecutive bifurcations have been observed and the obtained instability characteristics allowed to find the angular frequency of oscillations. For the higher Reynolds numbers the authors observed small-scale vortices near the inner cylinder similar to these presented in [5]. For the flow case $\Gamma=11.75, R_{m}=19$ the authors performed detailed computations for the symmetric and asymmetric and-wall boundary conditions and it has been found that the influence of the end-wall conditions on the flow structure for such high aspect ratio is small.

The investigations of the second flow case $\Gamma=3.76$, $R_{m}=2.2$ have allowed the authors to follow the transition from a three-cell structure to a one- cell structure which takes place with the gradually increasing Reynolds number in the case of asymmetric end-wall boundary conditions. This problem has been previously considered in [16-18], however, these investigations have been limited only to the low Reynolds numbers. The authors have analyzed the transition process from a three-cell structure to a one-cell structure and then, for higher $R e$, the development of the one-cell flow with the increasing Reynolds numbers. The results obtained for the higher Reynolds numbers have been compared with the result obtained experimentally within the frame of the EuHIT project in BTU in Cottbus.

The flow case $\Gamma=1.04, R_{m}=2.2$ with the asymmetric end-wall boundary conditions has also been investigated numerically and experimentally. An agreement between results has been found, however, a more detailed comparison between experimental and numerical results will be presented during upcoming conferences in Cottbus and Delft. The authors continue the numerical study of the flow cases $\Gamma=3.76$, $R_{m}=2.2, \Gamma=1.04, R_{m}=2.2$ for higher and higher $R e$, as well as, they continue investigations of the flow cases with heat transfer and with the oscillations superimposed on the rotational speed of the rotor.

\section{Acknowledgments}

The authors are grateful to the Poznan Supercomputing and Networking Center, where the computations have been performed, and EuHIT for founding the experimental investigation.

\section{References}

[1] K.T. Coughlin, P.S. Marcus, Modulated waves in TaylorCouette flow Part 1. Analysis. J. Fluid Mech. 234, 1-18 (1992).

[2] D.P. Lathrop, J. Fineberg, H.L. Swinney, Transition to sheardriven turbulence in Couette-Taylor flow. Phys. Rev. A 46, 6390-6405 (1992).

[3] Ch. Egbers, G. Pfister, Physics of rotating fluids, Lecture Notes in Physics. Springer (2000).

[4] O. Czarny, E. Serre, P. Bontoux, R.M. Lueptow, Interaction of wavy cylindrical Couette flow with end-walls. Phys. Fluids 16, 1140-1148 (2004).

[5] S. Dong, Direct numerical simulation of turbulent TaylorCouette flow. J. Fluid Mech. 587, 373 (2007).

[6] U. Harlander, G. Wright, Ch. Egbers, Reconstruction of the $3 D$ flow field in a differentially heated rotating annulus laboratory experiment. Geophysical Research Abstracts 14 EGU2012-5368 (2012).

[7] H. Brauckmann, B. Eckhardt, Direct numerical simulations of local and global torque in Taylor-Couette fow up to Re D 30000. J. Fluid Mech 718, 398 (2013).

[8] W. Serre, J.P. Pulicani, A three dimensional pseudo-spectral method for convection in rotating cylinder. J. Computers \& Fluids 30, 491 (2001).

[9] E. Tuliszka-Sznitko, A. Zieliński, W. Majchrowski, LES of the non-isothermal transition flow in rotating cavity, Int. J. Heat and Fluid Flow 30, 534 (2009).

[10] E. Tuliszka-Sznitko, A. Zieliński, W. Majchrowski, Large eddy simulation of non-isothermal flow in rotor/stator cavity. Proceedings of Int. Sym. On Heat Transfer in Gas Turbine Systems, Antalya, CD-ROM, pp.1-14, (2009).

[11] I.E. Tadmor, Convergence of spectral methods for nonlinear conservation laws. SIAM, J. Numerical Analysis, 26, 30 (1989).

[12] E. Severac, E. Serre, A spectral viscosity LES for the simulation of turbulent flows within rotating cavities. J. Comp. Phys. 226, 2, 1234 (2007).

[13] K. Kielczewski, E. Tuliszka-Sznitko, Numerical study of the flow structure and heat transfer in rotating cavity with and without jet. Arch. Mech. 65, 527 (2013).

[14] S. Sarra, Chebyshev Pseudospectral Methods for Conservation Laws with Source Terms and Application to Multiphase Flow. PhD Thesis, Morgantown, West Virginia (2002).

[15] K. Kiełczewski, E. Tuliszka-Sznitko, P. Bontoux, Numerical investigation of the Taylor-Couette and Batchelor flows with heat transfer: physics and numerical modeling. J. of Physics CS, 530, 1-8 (2014). 
[16] K.A. Cliffe, T. Mullin, A numerical and experimental study of anomalous modes in the Taylor experiment. J. Fluid Mech. 153, 243-258 (1985).

[17] B. Bansch, Ch. Egbers, O. Meincke, N. Scurtu, Taylor Couette System with Asymmetric Boundary Conditions. Universitat Bremen, Zentrum fur Thenomathematik, Report 0004 (2000).

[18] T. Mullin, C. Blohm, Bifurcation phenomena in a TaylorCouette flow with asymmetric boundary conditions. Phys. Fluids 13, 136 (2001).
[19] J. Jeong, F. Hussain, On the identification of a vortex. J. Fluid Mech. 285, 69 (1995).

[20] P. Chakraborty, S. Balachandar, R.J. Adrian, On the relationships between local vortex identification schemes. J. Fluid Mech. 535, 202 (2005).
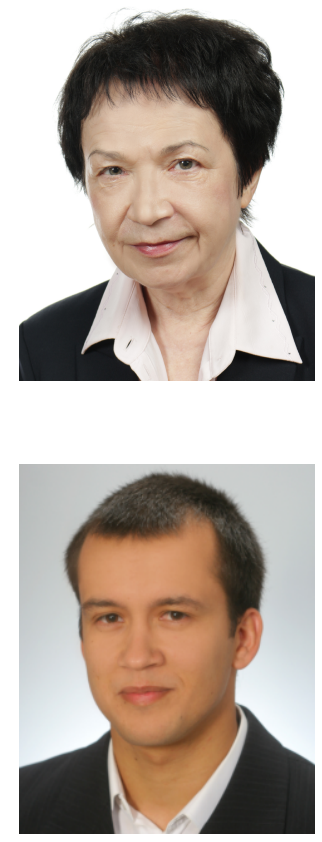

Ewa Tuliszka-Sznitko graduated from Poznan University of Technology, $\mathrm{PhD}$ in 1988, dr habil. in fluid mechanics in 1993 (Institute of Fluid Flow Machineries, Polish Academy of Sciences), professor title in 2012. Professor of Poznan University of Technology, Faculty of Working Machines and Transportation, Institute of Thermal Engineering. Author of about 120 papers published in international journals and presented during international congresses, and author of two books. Scientific visits and scholarships: University of Hanover, Arizona State University, Les Universites d'Aix-Marseille, Ecole Cental de Marseille. Research interests: computational fluid dynamics, instability, turbulence, heat transfer. Lecturer on the following subjects: thermodynamics, fluid mechanics, energy management.

Kamil Kiełczewski was born in Poznan in 1984. MSc in 2009 (Department of Mathematics and Computer Science, Adam Mickiewicz University, specialization: software engineering). $\mathrm{PhD}$ on "Parallel computing of flows in rotating disk system with cooling streams" in 2015 (Faculty of Working Machines and Transportation, Poznan University of Technology). Programing languages: Fortran, Java, C++, C\#, Ruby. The author of 15 papers. 